\title{
Growth and decay of localized disturbances on a surfactant-coated spreading film
}

\author{
Benjamin J. Fischer and Sandra M. Troian* \\ Department of Chemical Engineering, Princeton University, Princeton, New Jersey 08544-5263
}

(Received 8 March 2002; published 31 January 2003)

\begin{abstract}
If the surface of a quiescent thin liquid film is suddenly coated by a patch of surface active material like a surfactant monolayer, the film is set in motion and begins spreading. An insoluble surfactant will rapidly attempt to coat the entire surface of the film thereby minimizing the liquid's surface tension. The shear stress that develops during the spreading process produces a maximum in surface velocity in the region where the moving film meets the quiescent layer. This region is characterized by a shock front with large interfacial curvature and a corresponding local buildup of surfactant which creates a spike in the concentration gradient. In this paper, we investigate the sensitivity of this region to infinitesimal disturbances. Accordingly, we introduce a measure of disturbance amplification and transient growth analogous to a kinetic energy that couples variations in film thickness to the surfactant concentration. These variables undergo significant amplification during the brief period in which they are convected past the downstream tip of the monolayer, where the variation in concentration gradient and surface curvature are largest. Once they migrate past this sensitive area, the perturbations weaken considerably and the system approaches a stable configuration. It appears that the localized disturbances of the type we consider here, cannot sustain asymptotic instability. Nonetheless, our study of the dynamics leading to the large transient growth clearly illustrates how the coupling of Marangoni and capillary forces work in unison to stabilize the spreading process against localized perturbations.
\end{abstract}

DOI: 10.1103/PhysRevE.67.016309

PACS number(s): 47.20.Ma, 68.03.Cd, 68.15.+e, 47.85.Np

\section{INTRODUCTION}

Experimental studies have shown that the spontaneous spreading of a surfactant monolayer on a thin liquid film produces dendriticlike corrugations in the film thickness $[1-7]$. In situations where the spreading front is clearly visible, the dendritic patterns appear to form in the wake of the moving front. Plane projections of the fingered contours have been measured [8] to have a fractal dimension $D_{F} \sim 1.7 . \mathrm{Cu}-$ riously, this is the same fractal dimension measured in planar systems like viscous fingering in porous media or diffusion limited aggregation, both of which are governed by a time dependent diffusion equation. The mathematical relation between the equations governing these systems and the surfactant spreading problem, however, is not yet well understood. For example, the monolayer spreading problem is described by a coupled set of equations containing nonlinear diffusive terms (due to Marangoni convection) as well as higher order terms due to capillary forces, terms absent in the classic fingering equations. Despite these differences, the patterns formed assume shapes and fractal dimensions identical to those formed in strictly Laplacian-driven systems.

A time dependent model can describe the evolution of the film height and surfactant concentration containing Marangoni, capillary, and surface diffusion forces [9]. Because the constructed base states are fully time and space dependent, one must employ a fully transient analysis since any conclusions about the "stability" of the system to infinitesimal disturbances are only meaningful when compared to the evolution of the base state. In addition, the spatial dependence of the base-state film thickness $h_{o}$ and surface surfac-

\footnotetext{
*Electronic address: stroian@princeton.edu; URL: www.princeton.edu/ $\sim$ stroian
}

tant concentration $\Gamma_{o}$ gives rise to non-normal disturbance operators. As such, a conventional modal analysis of this system strictly captures only the asymptotic behavior of the spreading film as $\tau \rightarrow \infty$. This type of analysis might overlook significant transient excitations as first discussed by Ioannou and Farrell $[10,11]$ in the context of baroclinic instabilities. In this paper, we therefore present a linearized disturbance analysis of a thin liquid film driven to spread by Marangoni, capillary, and surface diffusion effects and focus on the transient behavior of disturbances localized at the leading edge.

In previous work [12-14], we quantified the level of disturbance amplification by introducing two energy norms, one associated with the perturbed film thickness $\widetilde{H}$ and the other with the surfactant concentration, $\widetilde{\Gamma}$. Both the optimal perturbations and the growth rate associated with disturbances in the transverse direction were computed. Similar behavior was reported whether the disturbances in the two variables were applied inphase or out of phase. In this paper, we resume this analysis but introduce instead a single energy norm directly related to the production of kinetic energy in the system. This single norm more easily identifies two key characteristics of the flow that dominate the large transient response, namely, the development of significant film curvature and the spike in the concentration gradient that develops at the advancing front of the spreading monolayer, where the base-state velocity is largest.

\section{TRANSIENT GROWTH ANALYSIS}

\section{A. Problem formulation}

\section{Base-state equations}

We first consider the one-dimensional spreading of an insoluble surfactant monolayer in the absence of disturbances. 


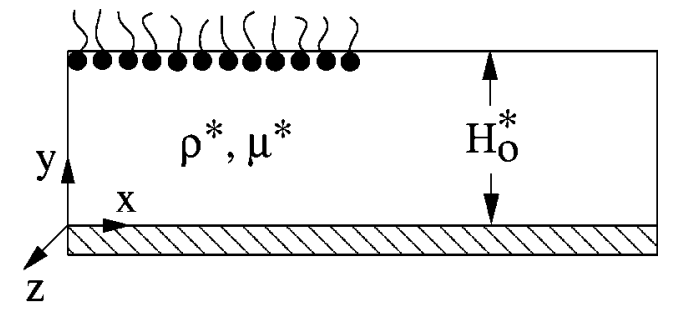

(a)

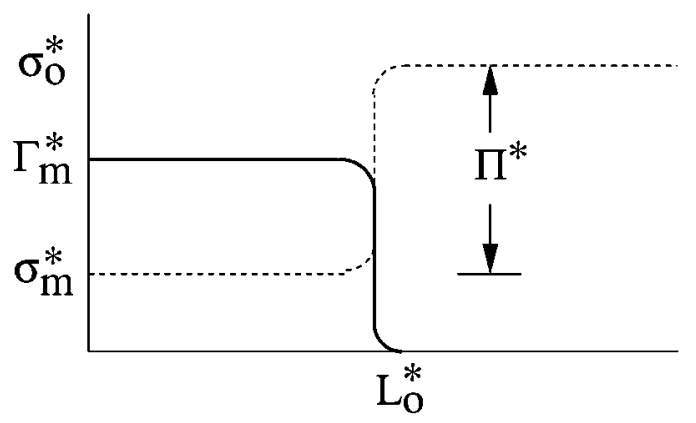

(b)

FIG. 1. The initial state of the Marangoni-driven spreading system. The liquid layer has a viscosity $\mu^{*}$, density $\rho^{*}$, and an initial uniform thickness $H_{o}^{*}$. Initially, the surfactant monolayer extends a distance $L_{o}^{*}$ with a surface tension $\sigma_{m}^{*}$ and surface concentration $\Gamma_{m}^{*}$. The uncontaminated liquid surface has a surface tension of $\sigma_{o}^{*}$, therefore, the maximum spreading pressure is $\Pi^{*}=\sigma_{o}^{*}$ $-\sigma_{m}^{*}$.

In Fig. 1 is shown a schematic diagram of the physical system. Utilizing the lubrication approximation and a linear equation of state relating surface tension to the surfactant surface concentration, the dimensionless evolution equations for the base-state film thickness $H_{o}$ and surface surfactant concentration $\Gamma_{o}$ in the limit of negligible Bond number, assume the form $[9,13]$

$$
\begin{gathered}
H_{o \tau}=\frac{1}{2}\left(H_{o}^{2} \Gamma_{o x}\right)_{x}-\frac{\mathcal{C}}{3}\left(H_{o}^{3} H_{o x x x}\right)_{x}, \\
\Gamma_{o \tau}=\left(\Gamma_{o} H_{o} \Gamma_{o x}\right)_{x}-\frac{\mathcal{C}}{2}\left(\Gamma_{o} H_{o}^{2} H_{o x x x}\right)_{x}+\frac{1}{\mathrm{Pe}_{s}} \Gamma_{o x x} .
\end{gathered}
$$

All subscripts $\tau$ and $x$ represent partial differentiation with respect to time or space. The dimensionless group $\mathcal{C}$ and the modified surface Peclet number $\mathrm{Pe}_{s}$, are defined by $\mathcal{C}$ $\equiv \varepsilon^{2} \sigma_{m}^{*} / \Pi^{*} \quad$ and $\quad \operatorname{Pe}_{s} \equiv\left(U^{*} L_{o}^{*}\right) / \mathcal{D}_{s}^{*}=\left(\Pi^{*} H_{o}^{*}\right) / \mu^{*} \mathcal{D}_{s}^{*}$, where $\varepsilon \equiv H_{o}^{*} / L_{o}^{*}$. $H_{o}^{*}$ represents the initial undisturbed film thickness, $L_{o}^{*}$ the initial extent of the monolayer, and $\mathcal{D}_{s}^{*}$ the surface diffusion coefficient of the surfactant on the liquid. The fluid is characterized by the viscosity $\mu^{*}$ and density $\rho^{*}$. The parameter $\mathcal{C}$ is related to the usual capillary number $\mathrm{Ca}=\mu^{*} U^{*} / \sigma_{m}^{*}$ through the relation $\mathcal{C}=\varepsilon^{3} / \mathrm{Ca}$. The maximal spreading pressure is defined by $\Pi^{*}=\sigma_{o}^{*}-\sigma_{m}^{*}$, where $\sigma_{o}^{*}$ is the surface tension of the clean liquid layer and $\sigma_{m}^{*}$ the initial surface tension of the monolayer coated film. The character- istic spreading velocity, dominated by Marangoni stresses, is denoted by $U^{*}=\varepsilon \Pi^{*} / \mu^{*}$. This velocity is typically orders of magnitude faster than the velocity induced by surface diffusion, as characterized by the surface diffusion coefficient $\mathcal{D}_{s}^{*}$. For large Peclet numbers, the term proportional to $\left(\mathrm{Pe}_{s}\right)^{-1}$ can be omitted altogether. The dimensionless time is scaled on the Maragoni velocity and the initial extent of the monolayer according to $L_{o}^{*} / U^{*}=\mu^{*} L_{o}^{* 2} / H_{o}^{*} \Pi^{*}$.

These equations are rescaled by introducing a self-similar variable [9] $\xi=x / L(\tau)$, which tracks the temporal evolution of the leading edge of the monolayer, $L(\tau)$ :

$$
H_{o}(x, \tau)=h_{o}(\xi, \tau) \text { and } \Gamma_{o}(x, \tau)=\frac{g_{o}(\xi, \tau)}{L(\tau)} .
$$

The additional factor of $L(\tau)$ that appears in the denominator of $\Gamma_{o}(x, \tau)$ is the result of imposing a finite mass of surfactant for surface distribution. The rate at which a monolayer advances over a liquid film depends on the geometry of spreading (rectilinear vs cylindrical) and whether the mass distributed from the surfactant reservoir is a constant or time dependent. A constant mass in rectilinear geometry produces an advancing monolayer front that grows in time as $[9,15]$ $L(\tau)=\tau^{1 / 3}$. These variable transformations reduce Eqs. (1) and (2) to

$$
\begin{aligned}
\tau h_{o \tau}= & \frac{1}{3} \xi h_{o \xi}+\frac{1}{2}\left(h_{o}^{2} g_{o \xi}\right)_{\xi}-\frac{\mathcal{C}}{3 \tau^{1 / 3}}\left(h_{o}^{3} h_{o \xi \xi \xi}\right)_{\xi}, \\
\tau g_{o \tau}= & \frac{1}{3}\left(\xi g_{o}\right)_{\xi}+\left(g_{o} h_{o} g_{o \xi}\right)_{\xi}-\frac{\mathcal{C}}{2 \tau^{1 / 3}}\left(g_{o} h_{o}^{2} h_{o \xi \xi \xi}\right)_{\xi} \\
& +\frac{\tau^{1 / 3}}{\mathrm{Pe}_{s}} g_{o \xi \xi} .
\end{aligned}
$$

Equations (4) and (5) are solved subject to the following boundary conditions:

$$
\begin{aligned}
& h_{o \xi}(0, \tau)=0, \quad h_{o \xi \xi \xi}(0, \tau)=0, \quad \text { and } \quad g_{o \xi}(0, \tau)=0 \text {, } \\
& h_{o}(\infty, \tau)=1, \quad h_{o \xi}(\infty, \tau)=0, \quad \text { and } \quad g_{o}(\infty, \tau)=0 .
\end{aligned}
$$

The condition (6) enforces symmetry and no-flux about the origin, while Eq. (7) enforces a quiescent and surfactant-free liquid film far downstream of the spreading monolayer.

The initial conditions $(\tau=1)$ chosen for this study correspond to an initially flat liquid film coated with a monolayer of insoluble surfactant of extent $2 L_{o}^{*}$ centered about the origin. The surfactant concentration is relatively flat and smoothly decays to zero near the point $\xi_{o}$. These two conditions are given by

$$
h_{0}(\xi, 1)=1
$$

and

$$
g_{o}(\xi, 1)=g_{o}^{\max }\left\{1-\tanh \left[\mathrm{A}\left(\xi-\xi_{o}\right)\right]\right\} .
$$




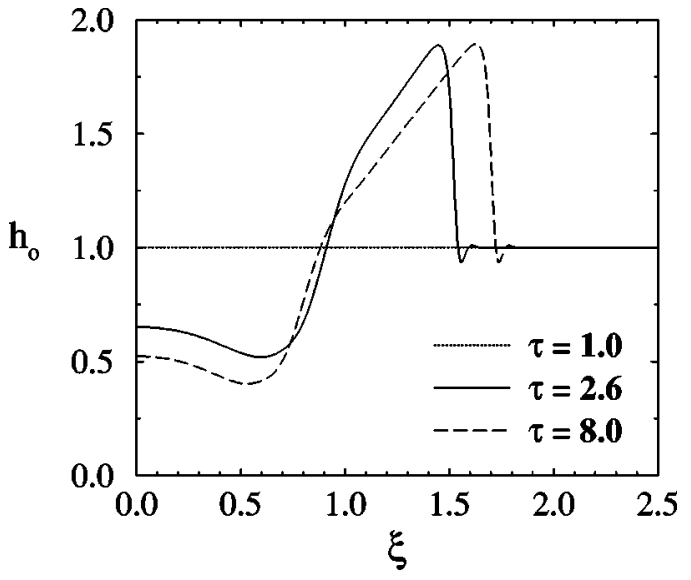

(a)

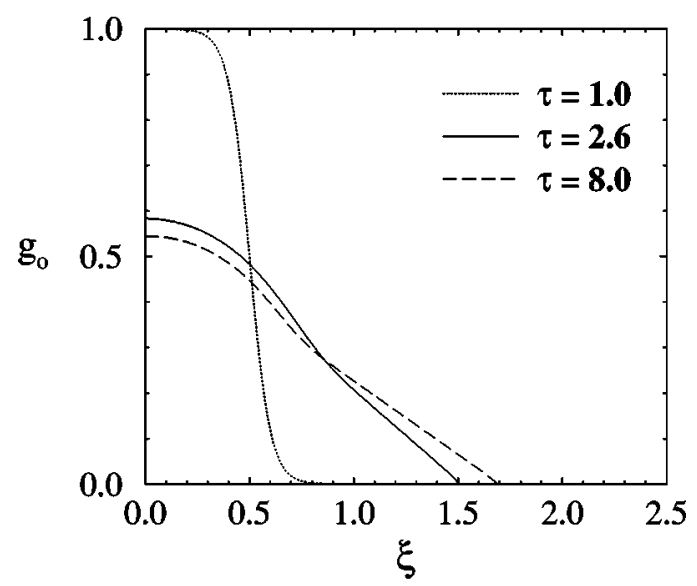

(b)

FIG. 2. Solutions for the base-state (a) film thickness $h_{o}$ and (b) surface surfactant concentration $g_{o}$ for times ranging from $1.0 \leqslant \tau$ $\leqslant 8.0$ with $\mathrm{Pe}_{s}=5000$ and $\mathcal{C}=10^{-5}$.

In this study, we held fixed the following parameter values: $\mathrm{g}_{\mathrm{o}} \max =0.5, A=10$ and $\xi_{o}=0.5$. Figure 2 shows $h_{o}$ and $g_{o}$ for times of $1.0 \leqslant \tau \leqslant 8.0$ when $\mathrm{Pe}_{s}=5000$ and $\mathcal{C}=10^{-5}$.

\section{Linearized disturbance equations}

In order to examine the stability of this system, perturbations are applied to both the height profile and surfactant surface concentration. Consequently, the total height and concentration profiles are

$$
h_{t o t}=H_{o}+\delta \tilde{H} \text { and } \Gamma_{t o t}=\Gamma_{o}+\delta \tilde{\Gamma} \text {, }
$$

where $\delta$ is a small parameter. The evolution of infinitesimal, two-dimensional disturbances, is governed by the pair of linearized equations [13]

$$
\begin{aligned}
\widetilde{H}_{\tau}= & \frac{1}{2}\left(H_{o}^{2} \widetilde{\Gamma}_{x}+2 H_{o} \Gamma_{o x} \widetilde{H}\right)_{x}+\frac{1}{2} H_{o}^{2} \widetilde{\Gamma}_{z z}-\frac{\mathcal{C}}{3}\left[\left(H_{o}^{3} \widetilde{H}_{x x x}\right.\right. \\
& \left.\left.+3 H_{o}^{2} H_{o x x x} \widetilde{H}\right)_{x}+\left(H_{o}^{3}\right)_{x} \widetilde{H}_{x z z}+2 H_{o}^{3} \widetilde{H}_{x x z z}+H_{o}^{3} \widetilde{H}_{z z z z}\right],
\end{aligned}
$$

$$
\begin{aligned}
\tilde{\Gamma}_{\tau}= & \left(\Gamma_{o} \Gamma_{o x} \widetilde{H}+H_{o} \Gamma_{o x} \widetilde{\Gamma}+\Gamma_{o} H_{o} \widetilde{\Gamma}_{x}\right)_{x}+\Gamma_{o} H_{o} \widetilde{\Gamma}_{z z} \\
& -\frac{\mathcal{C}}{2}\left[\left(\Gamma_{o} H_{o}^{2} \widetilde{H}_{x x x}+2 \Gamma_{o} H_{o} H_{o x x x} \widetilde{H}+H_{o}^{2} H_{o x x x} \widetilde{\Gamma}\right)_{x}\right. \\
& \left.-\left(\Gamma_{o} H_{o}^{2}\right)_{x} \widetilde{H}_{x z z}+2 \Gamma_{o} H_{o}^{2} \widetilde{H}_{x x z z}+\Gamma_{o} H_{o}^{2} \widetilde{H}_{z z z z}\right] \\
& +\frac{1}{\operatorname{Pe}_{s}}\left(\widetilde{\Gamma}_{x x}+\widetilde{\Gamma}_{z z}\right),
\end{aligned}
$$

where $x$ denotes the streamwise coordinate and $z$ the transverse coordinate. These dimensionless variables are scaled on the initial coverage length $L_{o}^{*}$. All disturbance variables are denoted by a tilde sign. Since each of the coefficients in this pair of equations depends only on the streamwise and not the transverse coordinate, the disturbance quantities can be Fourier decomposed according to

$$
(\widetilde{H}, \widetilde{\Gamma})(x, z, \tau)=(\Psi, \Phi)(x, \tau) e^{i K z},
$$

where $K$ defines the dimensionless transverse wave number of the associated disturbance. The Fourier amplitudes $\Psi$ and $\Phi$ can be rescaled to self-similar form as were the base states previously, according to

$$
\Psi(x, \tau)=\psi(\xi, \tau) \quad \text { and } \quad \Phi(x, \tau)=\frac{\phi(\xi, \tau)}{\tau^{1 / 3}}
$$

These transformations rescale Eqs. (10) and (11) to the form

$$
\begin{aligned}
\tau \psi_{\tau}= & \frac{1}{3} \xi \psi_{\xi}+\frac{1}{2}\left(h_{o}^{2} \phi_{\xi}+2 h_{o} g_{o \xi} \psi\right)_{\xi}-\frac{\left(K \tau^{1 / 3}\right)^{2}}{2} h_{o}^{2} \phi \\
& -\frac{\mathcal{C}}{3 \tau^{1 / 3}}\left\{\left(h_{o}^{3} \psi_{\xi \xi \xi}+3 h_{o}^{2} h_{o \xi \xi \xi} \psi\right)_{\xi}-\left(K \tau^{1 / 3}\right)^{2}\left[\left(h_{o}^{3}\right)_{\xi} \psi_{\xi}\right.\right. \\
& \left.\left.+2 h_{o}^{3} \psi_{\xi \xi}\right]+\left(K \tau^{1 / 3}\right)^{4} h_{o}^{3} \psi\right\}
\end{aligned}
$$

$$
\begin{aligned}
\tau \phi_{\tau}= & \frac{1}{3}(\xi \phi)_{\xi}+\left(g_{o} g_{o \xi} \psi+h_{o} g_{o \xi} \phi+h_{o} g_{o} \phi_{\xi}\right)_{\xi} \\
& -\left(K \tau^{1 / 3}\right)^{2} h_{o} g_{o} \phi-\frac{\mathcal{C}}{2 \tau^{1 / 3}}\left\{\left(g_{o} h_{o}^{2} \psi_{\xi \xi \xi}+2 g_{o} h_{o} h_{o \xi \xi \xi} \psi\right.\right. \\
& \left.+h_{o}^{2} h_{o \xi \xi \xi} \phi\right)_{\xi}-\left(K \tau^{1 / 3}\right)^{2}\left[\left(g_{o} h_{o}^{2}\right)_{\xi} \psi_{\xi}+2 g_{o} h_{o}^{2} \psi_{\xi \xi}\right] \\
& \left.+\left(K \tau^{1 / 3}\right)^{4} g_{o} h_{o}^{2} \psi\right\}+\frac{\tau^{1 / 3}}{\operatorname{Pe}_{s}}\left[\phi_{\xi \xi}-\left(K \tau^{1 / 3}\right)^{2} \phi\right] .
\end{aligned}
$$

The boundary conditions for the disturbance equations are given by

$$
\psi_{\xi}(0, \tau)=0, \quad \psi_{\xi \xi \xi}(0, \tau)=0, \quad \text { and } \quad \phi_{\xi}(0, \tau)=0 \text {, }
$$




$$
\psi(\infty, \tau)=0, \quad \psi_{\xi}(\infty, \tau)=0, \quad \text { and } \quad \phi(\infty, \tau)=0
$$

The same type of symmetry and decay boundary conditions applied to the base states are also applied to the disturbance functions.

The initial disturbances are Gaussian distributed about the point $\xi=\xi_{s}$. This location can be positioned in front of, behind, or at the monolayer front. The disturbances are described by

$$
\psi(\xi, 1)=\phi(\xi, 1)=e^{-\mathrm{B}\left(\xi-\xi_{s}\right)^{2}}
$$

The amplitude coefficients for $\psi(\xi, 1)$ and $\phi(\xi, 1)$ were chosen to be one since Eqs. (14) and (15) are already linearized. In this study, we held fixed the parameter value $B=50$.

According to Eq. (9), $\Gamma_{\text {tot }}$ may assume a negative value far downstream where $\Gamma_{o}=0$. The relevant variable in this system is the surface tension which is related to the concentration through the equation of state $\sigma=1-\Gamma$. Therefore, a negative concentration simply implies that the surface tension far downstream has been positively perturbed. This could be caused by external perturbations such as a local decrease in temperature or other surface heterogenities in the liquid film.

\section{B. Quantifiers of transient amplification}

Quantification of the growth or decay of a disturbance must be carefully monitored when applied to a time dependent base state. A convenient measure is the relative kinetic energy contained in the disturbance, $E_{d}(\tau)$, to that contained in the reference base state, $E_{b}(\tau)$ at time $\tau$. This relative energy is normalized by the initial (relative) input energy at time $\tau_{o}$, which defines the amplification factor $G$ according to

$$
G=\left[\frac{E_{d}(\tau)}{E_{d}\left(\tau_{o}\right)}\right] /\left[\frac{E_{b}(\tau)}{E_{b}\left(\tau_{o}\right)}\right]
$$

The amplification ratio $G$ describes how the relative input energy intensifies or dissipates in time.

This measure of amplification or decay can be used to identify the "momentary stability" of the spreading system [16] by considering the normalized rate of growth of disturbances given by

$$
\Omega \equiv \frac{1}{G} \frac{d G}{d \tau}
$$

The system displays momentary stability if $\Omega<0$ and momentary instability if $\Omega>0$. Systems for which $\Omega$ approaches a negative value at $\tau \rightarrow \infty$ are asymptotically stable.

In previous work, we considered two separate measures of mechanical energy, each associated with the disturbance functions $\psi(\xi, \tau)$ and $\phi(\xi, \tau)$ [13]. The solutions for $\psi$ and $\phi$ were simultaneously solved from Eqs. (14) and (15) and used to define the mechanical energies associated with the base and disturbance states

$$
E_{q} \equiv \frac{1}{2} \int_{0}^{\infty} q^{2}(\xi, \tau) d \xi, \quad \text { where } \quad q=\psi, \phi, h_{o}, g_{o}
$$

There are two disadvantages to this approach. The energy defined in this way is not a kinetic energy per se but just a measure of the amplitude squares of the relevant functions. Second, the definitions introduce two separate amplification ratios and therefore two growth rates, one associated with variations in film thickness and the other with variations in the surfactant concentration. This separation of terms makes it difficult to trace the overall response of the system to an applied perturbation. Because the film thickness and surfactant concentration are coupled variables that both determine the spreading velocity, it is physically more appealing to consider a single measure of amplification and growth associated with the actual kinetic energy contained in the flow. This type of energy probe also provides a more direct means of isolating the factors responsible for large transient growth as discussed in Sec. III. We therefore introduce the following quantifier of amplification, namely:

$$
\begin{gathered}
E_{b} \equiv \frac{1}{2 \lambda} \int_{0}^{\lambda} \int_{0}^{\infty}\left|\left\langle v_{o}\right\rangle\right|^{2}(\xi, \tau) d \xi d z, \\
E_{d} \equiv \frac{1}{2 \lambda} \int_{0}^{\lambda} \int_{0}^{\infty}|\langle\tilde{v}\rangle|^{2}(\xi, \tau) d \xi d z .
\end{gathered}
$$

The subscripts " $b$ " and " $d$ " denote the base state and disturbance, respectively, and the dimensionless, transverse disturbance wavelength is denoted by $\lambda=2 \pi / K$. The kinetic energy per unit wavelength in the transverse direction, $E_{j}$, contained in the flow is found by averaging the velocity squared over the film thickness (i.e., $\langle\cdot\rangle$ ). The magnitude of the base-state velocity is denoted by $\left|\left\langle v_{o}\right\rangle\right|$ and that of the disturbance velocity by $|\langle\tilde{v}\rangle|$. The components of the heightaveraged base-state velocity in the streamwise and transverse directions are given by

$$
\begin{gathered}
\left\langle u_{o}\right\rangle=-\frac{1}{2 \tau^{2 / 3}} h_{o} g_{o \xi}+\frac{\mathcal{C}}{3 \tau} h_{o}^{2} h_{o \xi \xi \xi}, \\
\left\langle w_{o}\right\rangle=0,
\end{gathered}
$$

respectively, while those of the averaged disturbance velocities are given by

$$
\begin{aligned}
\langle\tilde{u}\rangle= & {\left[-\frac{1}{2 \tau^{2 / 3}}\left(h_{o} \phi_{\xi}+g_{o \xi} \psi\right)+\frac{\mathcal{C}}{3 \tau} h_{o}\left(h_{o} \psi_{\xi \xi \xi}+2 h_{o \xi \xi \xi} \psi\right.\right.} \\
& \left.\left.-\tau^{2 / 3} K^{2} h_{o} \psi_{\xi}\right)\right] e^{i K z}, \\
\langle\tilde{w}\rangle= & {\left[-\frac{1}{2 \tau^{1 / 3}} K h_{o} \phi+\frac{\mathcal{C}}{3 \tau^{2 / 3}} K h_{o}^{2}\left(\psi_{\xi \xi}-\tau^{2 / 3} K^{2} \psi\right)\right] i e^{i K z} . }
\end{aligned}
$$


As time advances, the base-state height and concentration profiles approach self-similar profiles in $\xi$ [9]. Therefore, at long times, the dominant time-dependence in Eq. (23) comes from the explicit $\tau$ dependence. At late times, $u_{o}$ will be dominated by the first term in Eq. (23) since $\tau^{-2 / 3}$ decreases slower than $\tau^{-1}$ and $\mathcal{C}$ is a small number. Consequently, the base-state energy should decrease with a $\tau^{-4 / 3}$ dependence. Although not shown, this result was numerically confirmed.

Therefore, in order to observe an unstable system, the disturbance energy will have to decrease slower than a $\tau^{-4 / 3}$ dependence. Since, we expect the system energy to follow a power-law behavior in time, it will be useful to plot the amplification factor-time data on a log-log plot. Similarly, the growth rate should be compared with $1 / \tau$.

\section{Numerical procedure}

The four equations (4), (5), (14), and (15) were simultaneously solved by the method of lines [17], which implements second-order centered differences for the spatial derivatives and a fully implicit Gear's method for the time integration [18]. At the start of each simulation, the dimensionless parameters $\mathcal{C}, \mathrm{Pe}_{s}$, and $K$ were specified. In this work, we held fixed the value of $\mathcal{C}$ at $10^{-5}$ (which gives prominence to Marangoni stresses) and used two values of the Peclet number, namely, 100 and 5000 [19]. More importantly, we varied the position of the initial disturbance, its initial shape (including the height and surfactant distribution), and the disturbance wave number. Depending on the initial location of the disturbances, the number of grid points used in the computations varied between 301 and 751 .

\section{RESULTS AND DISCUSSION}

\section{A. Numerical results}

\section{Disturbances localized at the monolayer edge}

The initial surfactant distribution $g_{o}$ tails off at $\xi_{o}=0.5$ and completely vanishes around $\xi=0.8$ [see Fig. 2(b)] according to Eq. (8). When disturbances are applied near the base of the concentration decay point, at $\xi_{s}=0.7$, the system undergoes maximum amplification for the lowest wave numbers, as shown in Fig. 3(a). The overall maximum occurs for $K=0$; the larger the disturbance wave number, the smaller the amplification obtained. The modes $K \neq 0$ exhibit a short period of amplification for $\tau<1.3$ but rapidly decay to zero. As shown in Fig. 3(b), the growth rate for each mode, $\Omega$, is positive over an even smaller interval of time $1.0<\tau<1.2$. The $K=10$ mode is somewhat different in that it displays a second small growth spurt about $\tau=1.2$ before decaying to zero like the rest. Each mode displays a momentary stabilizing response when the disturbance is first applied. We interpret this to be the system's attempt to induce Marangoni flows in the transverse direction that momentarily weaken the streamwise flow. However, the system overshoots this response and causes a slightly enhanced streamwise flow which leads to the global maximum in $\Omega$ shown for each curve. Eventually, of course, because the mass of surfactant

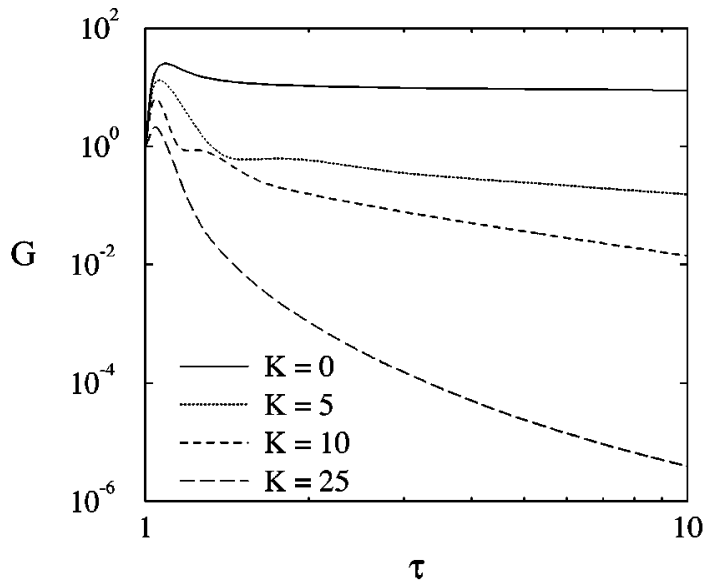

(a)

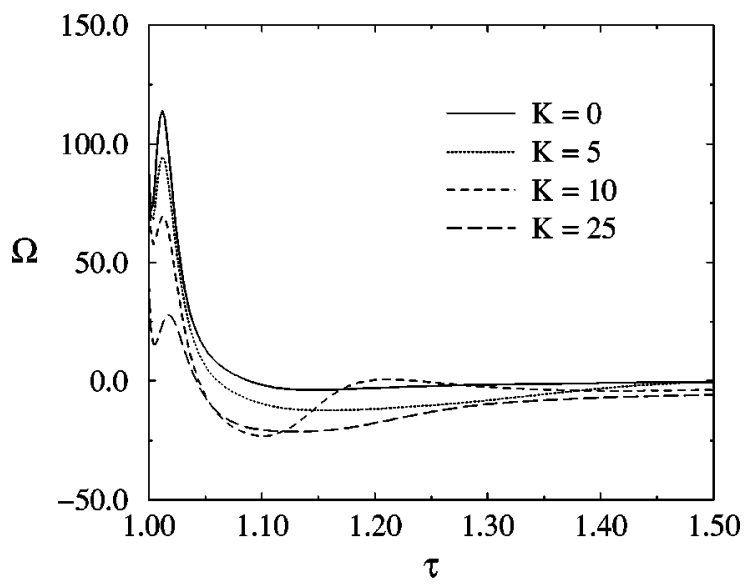

(b)

FIG. 3. Time evolution of the (a) amplification ratio and (b) normalized rate of energy growth for $\xi_{s}=0.7$ and disturbance wave numbers in the range $0 \leqslant K \leqslant 25$. Other parameter values are specified in the text.

distributed is finite, the overall driving force for spreading continually decreases and the perturbations dampen and vanish as $\tau \rightarrow \infty$.

\section{Disturbance localized far downstream}

When the initial disturbance is applied at $\xi_{s}=2.0$, much further in front of the concentration decay point, more substantial amplification is achieved as shown in Fig. 4(a). The disturbance amplification occurs at later times $\tau>2.0$, since the spreading front must advance close to $\xi_{s}=2.0$ to sense the disturbance. Not only are the amplitudes an order of magnitude larger than the first case discussed above, but the "interaction times" also last much longer. This behavior can be traced to the fact that the speed of the advancing front decays in time as $d L / d \tau \sim \tau^{-2 / 3}$. The disturbance, therefore, has a longer residence time in the area of the shocklike front that develops at the surfactant leading edge (typical profiles of the liquid film at three different times are shown in Fig. 2). Also, when a disturbance was applied at the base of the concentration decay point, the $K=0$ mode underwent the largest overall amplification. In contrast, placing the distur- 


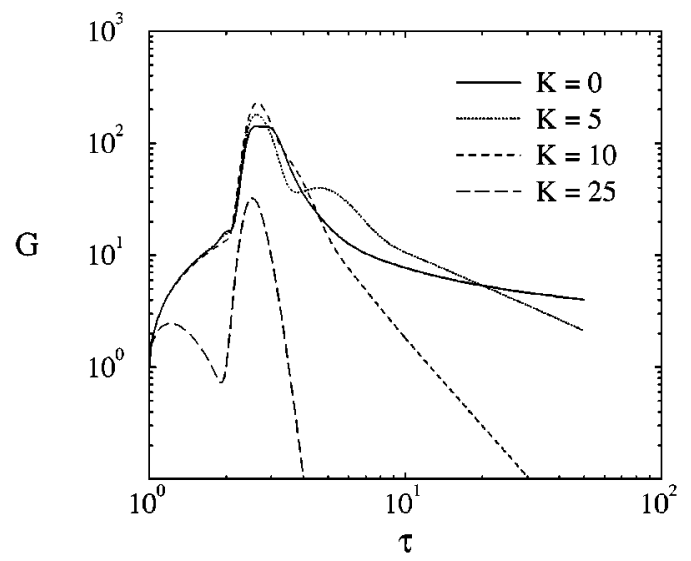

(a)

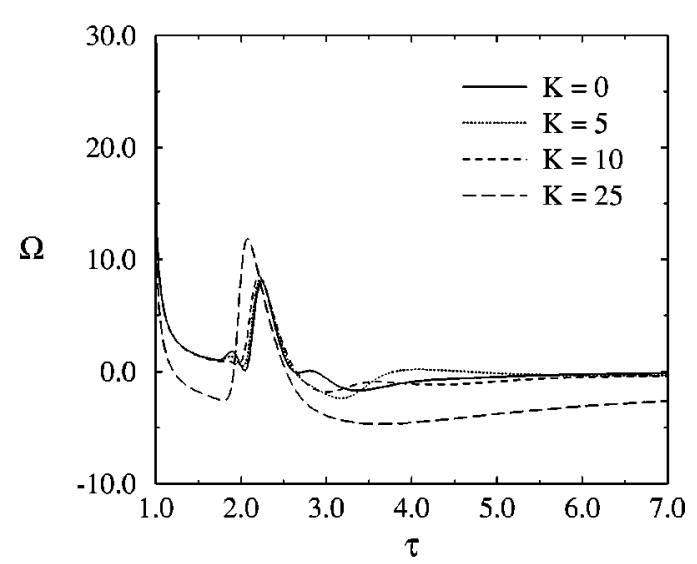

(b)

FIG. 4. Time evolution of the (a) amplification ratio and (b) normalized rate of energy growth for $\xi_{s}=2.0$ and disturbance wave numbers in the range $0 \leqslant K \leqslant 25$. Other parameter values are specified in the text.

bance further ahead of this point and allowing the film profile time to develop a significant shocklike front, switches the maximum amplification amplitude to the mode $K=10$, with $K=5,0$, and 25 undergoing progressively smaller intensification, in that order. The largest wave number tried, $K$ $=25$, shows insignificant perturbation enhancement but displays the largest overall growth rate as shown in Fig. 4(b). Another interesting difference related to disturbance localization further ahead of the initial surfactant distribution lies with the time of onset of amplification. If the front is allowed time to evolve before merging with the perturbation, all wave numbers are excited and reach their peak at approximately the same time, in contrast to the first case discussed in Fig. 3, in which the smaller wave numbers take longer to assume their maximum value. The growth rate curves for $\xi_{s}=2.0$ shown in Fig. 4(b) are similar to those shown previously for $\xi_{s}=0.7$ except for the delayed response corresponding to the time required for the spreading front to meet the applied disturbance.

\section{Disturbances localized upstream of the monolayer edge}

When the disturbances $\phi$ and $\psi$ are applied well inside the initial surfactant distribution, for example, at $\xi_{s}=0.4$,

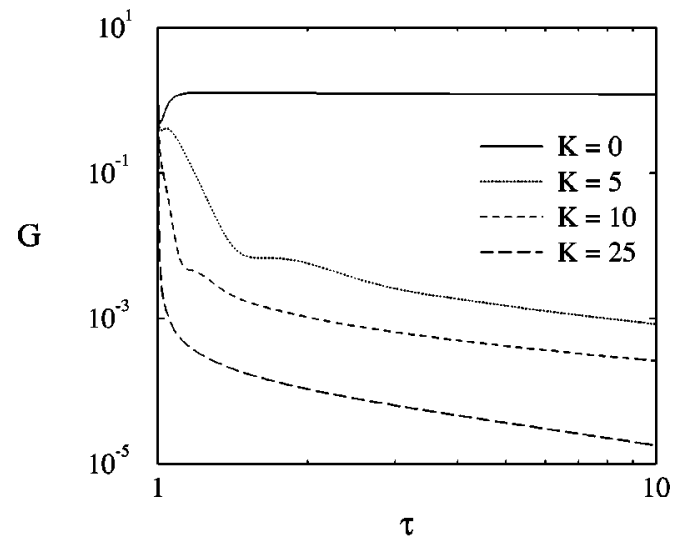

(a)

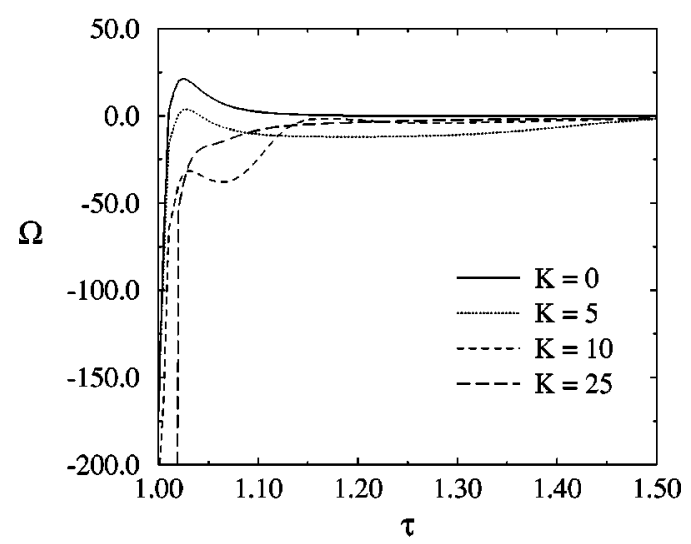

(b)

FIG. 5. Time evolution of the (a) amplification ratio and (b) normalized rate of energy growth for $\xi_{s}=0.4$ and disturbance wave numbers in the range $0 \leqslant K \leqslant 25$. Other parameter values are specified in the text.

there is no transient amplification or growth. Figure 5(a) shows that for all modes except $K=0$, the amplification rapidly decays from unity to zero. Although the $K=0$ mode also shows little if any amplitude intensification, it does require a much longer time to decay to zero. The growth rate curves corresponding to this system are depicted in Fig. 5(b). Unlike the two previous simulations, there is no sudden stabilizing response as the disturbances are first applied to the spreading film. This is likely due to the fact that the disturbances are applied well inside the initial surfactant monolayer where the film profile is still rather flat and uniform.

In Table I, the position of the applied disturbance is listed along with the mode undergoing the largest overall amplification. For disturbances initially located within the region coated by surfactant or close to the point where the initial surfactant concentration decays to zero $\left(\xi_{o} \approx 0.5\right)$, the $K$ $=0$ mode exhibits the largest amplification and growth rate. If the initial disturbances are applied well beyond the surfactant concentration decay point, the $K=10$ mode exhibits the largest amplification ratio and growth rate. We did not investigate the amplification ratios for smaller increments in wave number but the choice $K=11$, for example, gave an amplification ratio profile similar to the $K=10$ mode for $\xi_{s}$ $=1.5,1.7$, and 2.0. Also, when decreasing $\mathrm{Pe}_{s}$ to 500 , the 
TABLE I. Wave numbers corresponding to the applied disturbance yielding the largest amplification ratio. All relevant parameters are held fixed except the location of the peak of the Gaussian distributed perturbation which varies between $0.0 \leqslant \xi_{s} \leqslant 2.0$. The relevant parameter values are $\mathrm{g}_{o}^{\max }=0.5, A=10, \xi_{o}=0.5, B=50$, $\mathrm{Pe}_{s}=5000$, and $\mathcal{C}=10^{-5}$. Parameter definitions can be found in Sec. II C.

\begin{tabular}{cc}
\hline \hline$\xi_{s}=0.0$ & $K_{\max }=0.0$ \\
\hline 0.4 & 0 \\
0.7 & 0 \\
1.0 & 0 \\
1.5 & 10 \\
1.7 & 10 \\
2.0 & 10 \\
\hline \hline
\end{tabular}

optimally growing mode shifted to the value $K=7$ for $\xi_{s}$ $=2$.0. It appears that there is some sort of preferred wavelength for maximal transient growth depending on the position of the initial disturbance and how well developed is the profile of the advancing shock in film thickness and surfactant concentration. Because $\operatorname{Pe}_{s}=\left(\Pi^{*} H_{o}^{*}\right) / \mu^{*} \mathcal{D}_{s}^{*}$, it is trivial to alter the magnitude of the parameter with various experimental conditions. Using dip-coating or spin-coating methods, a known thickness of liquid can be deposited on a substrate thereby altering $H_{o}^{*}$. Similarly, with different combinations of liquids and surfactants, the values of $\Pi^{*}, \mu^{*}$, and $\mathcal{D}_{s}^{*}$ can be modified to attain a desired $\mathrm{Pe}_{s}$. These trials can be attempted with manually applied disturbances at various locations by contacting a surfactant-coated wire to the surface of the liquid. Therefore, we can conceive of experiments that alter $\mathrm{Pe}_{s}$ and the initial disturbance location, while measuring the resulting finger wavelength.

\section{B. Enhancement of a localized disturbance}

A comparison of the disturbance functions, $\psi(\xi, \tau)$ and $\phi(\xi, \tau)$ for $K=10$ shown in Fig. 6, with the base state profiles, $h_{o}, g_{o}$, and $g_{o \xi}$, shown in Figs. 2 and 7, confirms that the largest transient response occurs when the disturbances are momentarily centered about the point where the surfactant concentration decays to zero. For the particular simulations shown, this occurs at $\xi=1.5$ for $\tau=2.6$. At this same location, the steep front of the advancing shock in $h_{o}$ undergoes an inflection in the slope. More importantly, the overall base state surface velocity achieves a maximum. Examination of the evolution of the gradient in the base-state surfactant concentration, $g_{o \xi}$, as seen in Fig. 7(a), indicates a strong kink at the local minimum for $\xi=1.5$. The quantity $g_{o \xi \xi}$ suffers the largest change at this location. This kink is also reflected in the plot of the base-state surface velocity shown in Fig. 7(b), where the velocity has been decomposed into the two main components of the flow, namely, the Marangoni and capillary contributions. The Marangoni contribution experiences a slight enhancement at $\xi=1.5$ which is directly counteracted by the negative capillary velocity. In fact, the larger $\mathrm{Pe}_{s}$ and the smaller $\mathcal{C}$, the stronger the re-

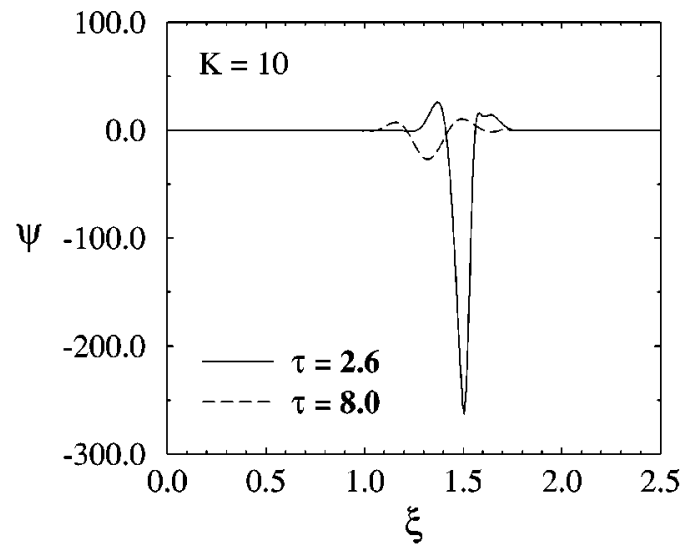

(a)

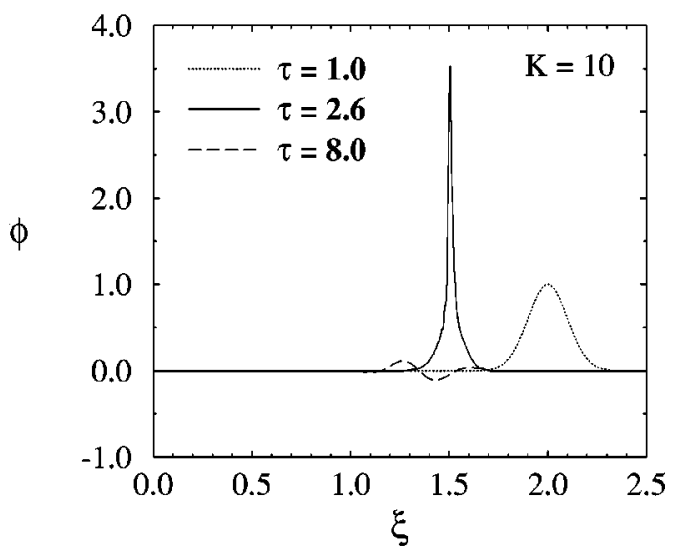

(b)

FIG. 6. Solutions for the disturbances in (a) thickness $\psi$ and (b) surfactant concentration $\phi$ for times ranging from $1.0 \leqslant \tau \leqslant 8.0$ with $K=10$ and $\xi_{s}=2.0$. The amplitude of the function $\psi(\xi, \tau=1)$ in (a) is too small to be visible on the scale shown.

sponse and counter-response. We discuss below the importance of this sharp variation and increase in $g_{o \xi}$.

As time evolves to $\tau=8.0$, the disturbances advect through the advancing shock region and fall behind the leading edge. A comparison of Fig. 2(a), Fig. 6(a), and Fig. 7 shows that the disturbances are now localized in the linear portion of the height profile where the concentration gradient is a constant. Once the disturbances fall behind the shock region in the base state, the amplitudes sharply decay and the momentary amplification is minimal. This trend continues until the amplitude decays to zero.

Our studies also confirm that as the wave number of the disturbances is increased beyond $K=10$, the lifetime of the applied perturbations rapidly decreases. For $K=25$, the amplitudes vanish before the disturbances have migrated to the linear portion of the base-state thickness profile. In addition, the oscillation seen previously in $\psi$ for the case $K=10$ completely disappears and instead a single peak appears centered at the point of maximum surface velocity. In the other limit, where $K=0$, the disturbances undergo significant amplification, as previously shown in Fig. 4. Interestingly, these disturbances $\psi$ and $\phi$ assume almost identical shape to the respective base-state functions $h_{o \xi}$ and $g_{o \xi}$. It was not possible 


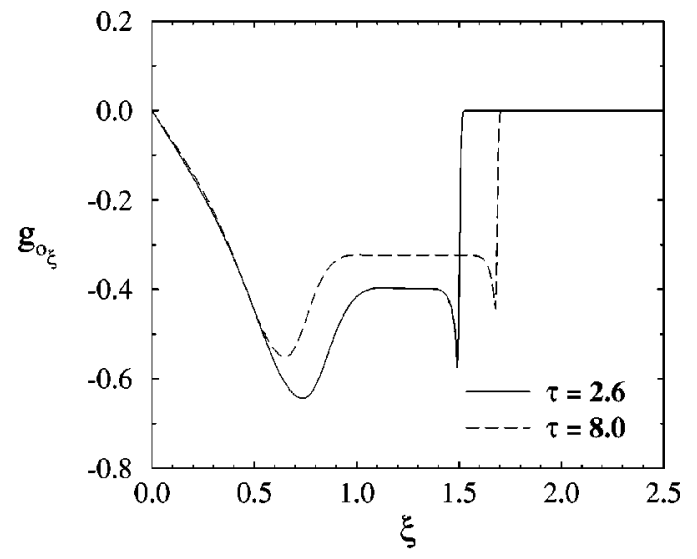

(a)

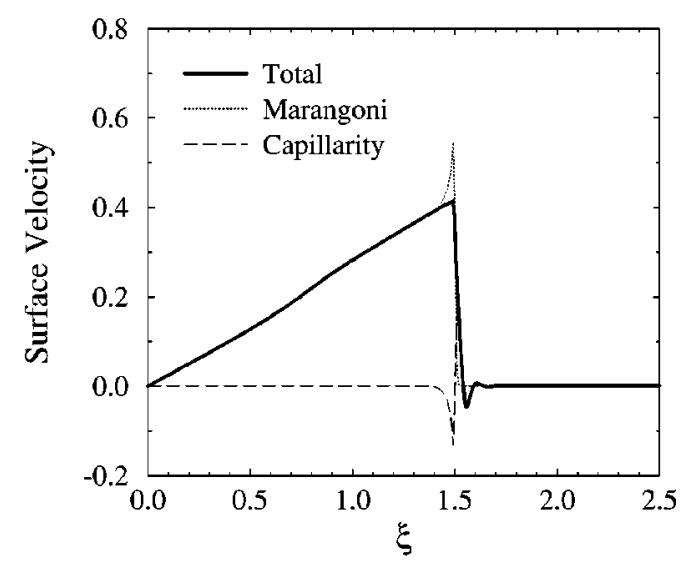

(b)

FIG. 7. (a) Comparison of the gradient in the base-state concentration profile, $g_{o \xi}$, for times $\tau=2.6$ and 8.0. A spike develops in the concentration profile, where $g_{o \xi}$ undergoes a steep increase to zero. (b) The Marangoni and capillary contributions to the basestate surface velocity profile, along with their sum at time $\tau=2.6$. Note the negative surface velocity just ahead of the step profile.

to derive this observation analytically from Eqs. (14) and (15).

These observations seem to suggest that disturbances which migrate and localize to the point of maximal surface velocity in the spreading film undergo significant amplification while "riding the wave" shown in Fig. 2(a). Once the perturbation falls behind the rapidly moving front, its amplitude continually decreases until it vanishes altogether. The exact point of maximum disturbance intensification always coincides with the point where the profile of the base-state surfactant concentration vanishes to zero.

\section{Effect of the initial surfactant distribution}

Because the transient amplification is correlated to the vanishing point in $g_{o}$, we wanted to study further what effect would result by smoothing out the kink in $g_{o \xi}$ shown in Fig. 7(a). Choosing an initial concentration profile with a more gradual decline to zero would lend insight into the dynamics of disturbance growth at the leading edge. An exponential type decay provides a more gradual decline than a hyperbolic tangent function. We therefore examined the behavior of the system for an initial deposition profile of the form

$$
g_{o}(\xi, 1)=m_{o} e^{-4 \pi \xi^{2}}
$$

To directly compare the evolution behavior of this distribution with the previous hyperbolic tangent form, it is important to maintain the same overall mass of surfactant deposited. A simple mass balance shows that this can be achieved with the choice $m_{o}=2.0$ in Eq. (25) for the previous choice $\mathrm{g}_{o}^{\max }=0.5$ and $A=10$ in Eq. (8).

Under the same conditions specified earlier, the exponential profile also produces large transient disturbance amplification and growth as shown in Fig. 8. In fact, the amplification ratio with the exponential deposition profile is significantly larger than that achieved with the hyperbolic tangent distribution studied earlier. The normalized growth rates are identical, however, and reproduce the general behavior seen previously in Fig. 4(b). This difference in amplification ratio is simply due to the larger kinetic energy input to the system initialized with the hyperbolic tangent distribution. Since the amplification ratio was defined by normalizing the momentary kinetic energy by its initial input value [see Eq. (19)], the system with the tanh deposition profile has a larger denominator throughout and therefore exhibits smaller disturbance intensification. As before, the disturbances with the smaller wave number $(K=10)$ undergo much stronger global amplification.

Comparison of the base-state velocity profiles for the exponential or tanh concentration distribution (not shown) depicts no difference. The peak in the disturbance profiles once again occurs at the point where the front of the advancing shock in $h_{o}$ undergoes an inflection, which corresponds to the point where $g_{o \xi}$ exhibits a kink as the concentration gradient rapidly increases from a negative value to zero. This result verifies that smoothing the rate of decay of the initial concentration profile by switching from a tanh to an exponential type distribution does not significantly dampen the amplification experienced by disturbances, which localize to this sensitive region of the flow.

\section{Effect of $\mathrm{Pe}_{\boldsymbol{s}}$}

Decreasing $\mathrm{Pe}_{s}$ from 5000 to 100 , leaving the initial concentration profile and all other relevant variables fixed ( $\mathrm{g}_{o}^{\max }=0.5, A=10, \xi_{o}=0.5, B=50$, and $\mathcal{C}=10^{-5}$ ) further smooths the height and concentration profile at the leading edge. Shown in Fig. 9(a) is a comparison of the base-state gradient, $g_{o \xi}$, with the original tanh mass distribution for two values of $\mathrm{Pe}_{s}$ at time $\tau=2.6$. Along with a smoother shock front (not shown), the kink in $g_{o \xi}$ is completely removed, leading to a strong decrease in the maximum disturbance amplitude from $\approx 230$ to 15 .

\section{E. Development of the surfactant distribution}

What leads to the sharp kink in $g_{o \xi}$ as the base-state concentration evolves and why is this region so sensitive to disturbances, amplifying infinitesimal perturbations by over two 


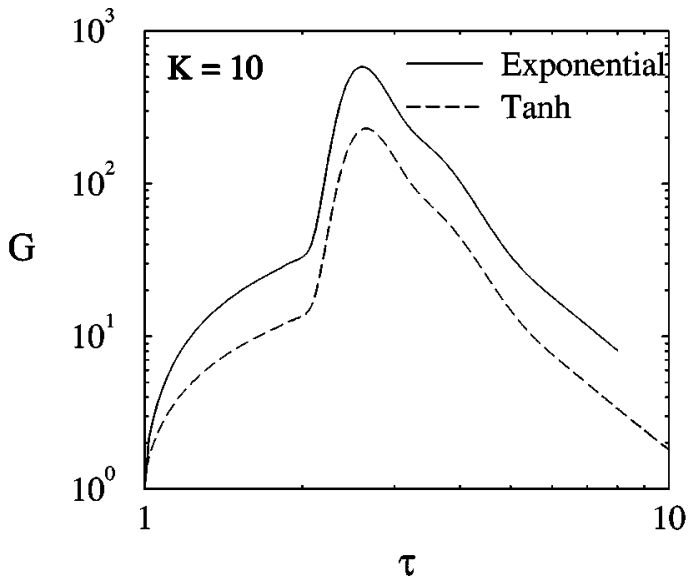

(a)

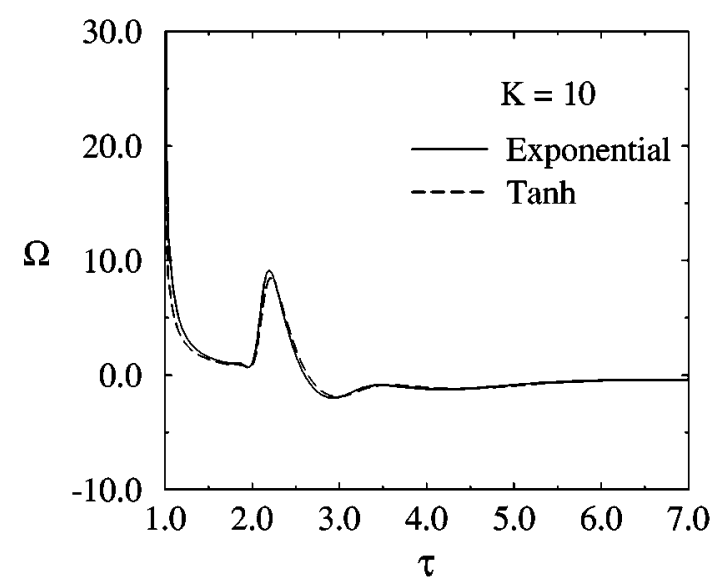

(c)

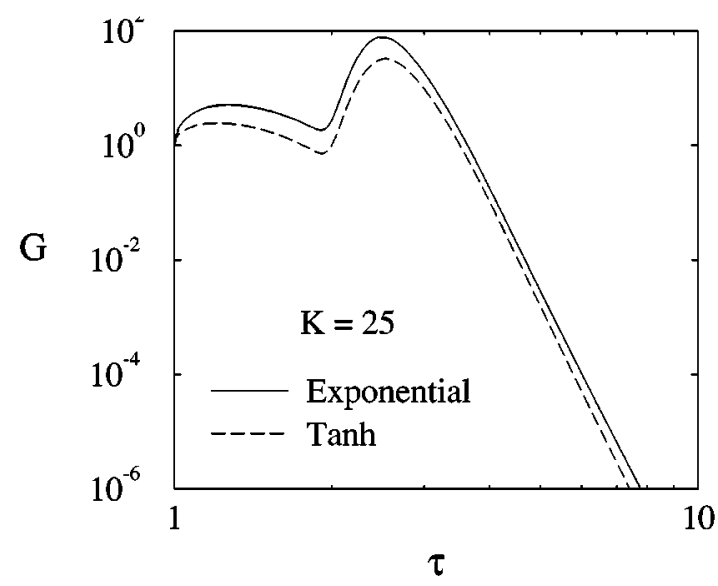

(b)

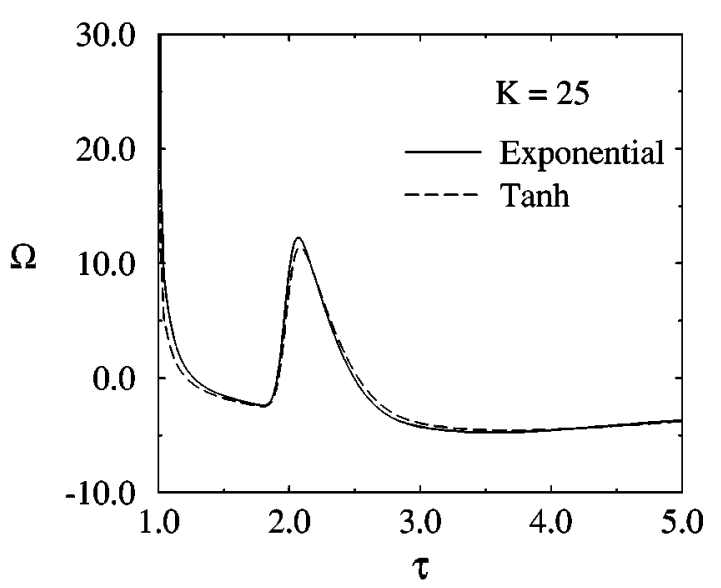

(d)

FIG. 8. Comparison of the amplification and growth of disturbances for two different initial surfactant concentration profiles (exponential vs hyperbolic tangent decay) with disturbances centered at $\xi_{s}=2.0$. (a) and (b): Amplification ratio for $K=10$ and $K=25$. (c) and (d) Normalized rate of energy growth for $K=10$ and $K=25$.

orders of magnitude? Returning to Fig. 9(a), the kink for $\mathrm{Pe}_{s}=5000$ at $\tau=2.6$ reflects the fact that the concentration gradient is fairly constant from $\xi=1.0$ to 1.5 and then suddenly jumps to a lower value before completely dying away. This sudden decrease implies that the base-state surfactant concentration undergoes a sudden decrease in slope at the downstream tip. Recall that the overall surface velocity shown in Fig. 7(b) corresponding to this same position and time achieves a small negative value just ahead of $\xi=1.5$. This negative velocity is due to a capillary pressure at the leading edge that tends to force the fluid upstream. Directly in front of this region, Marangoni and capillary mechanisms are trying to force fluid downstream. At the junction where the positive and negative surface velocities meet, surfactant has the propensity to buildup and thereby create the sharp kink in the gradient profile. If the level of surface diffusion is increased, then both the height and concentration profile smooth out at the leading edge. This lowers the capillary pressure at the front and significantly reduces the magnitude of the negative surface velocity. Therefore, the buildup of surfactant does not occur and the kink in the gradient is significantly reduced. As a result, the transient growth is substantially diminished. It is well known that there exists a singularity in the stress profile for the case where surface diffusion is completely absent caused by the junction of two surface profiles, one of which is stress laden (i.e., coated with surfactant) and the other stress-free (i.e., surfactant-free) $[20,21]$. The kink we have traced to the large transient growth is not related to this singularity in the flow since in our simulations there is always surface diffusion present.

\section{F. Surfactant equation of state}

All of the results noted above were obtained with the linear equation of state $\sigma=1-\Gamma$, where $\sigma$ and $\Gamma$ are the dimensionless surface tension and surfactant concentration, respectively. We also studied the behavior of a spreading surfactant monolayer described by a nonlinear equation of state [22]:

$$
\sigma(\Gamma)=(\beta+1)[1+\theta(\beta) \Gamma]^{-3}-\beta,
$$

where $\theta(\beta)=[(\beta+1) / \beta]^{1 / 3}-1$. A Taylor expansion of this expression for $\beta \rightarrow \infty$ reproduces the linear equation of state used in deriving the dimensionless pair of Eqs. (1) and (2). Small values of $\beta$ produce surfactant monolayers that are 


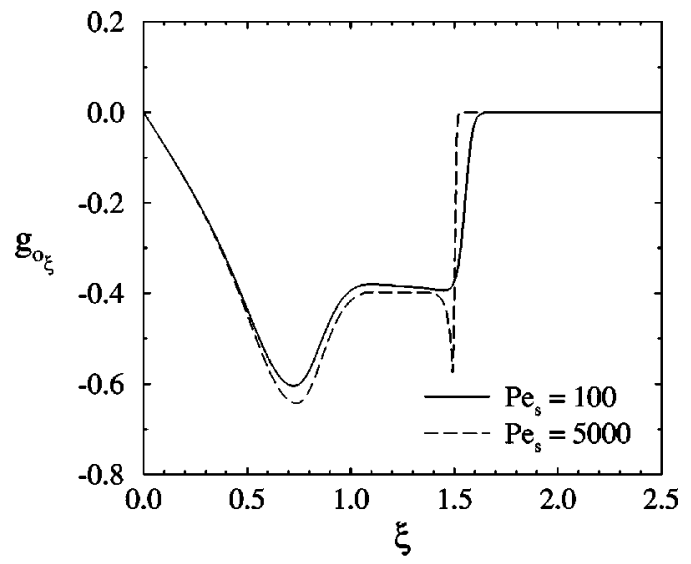

(a)

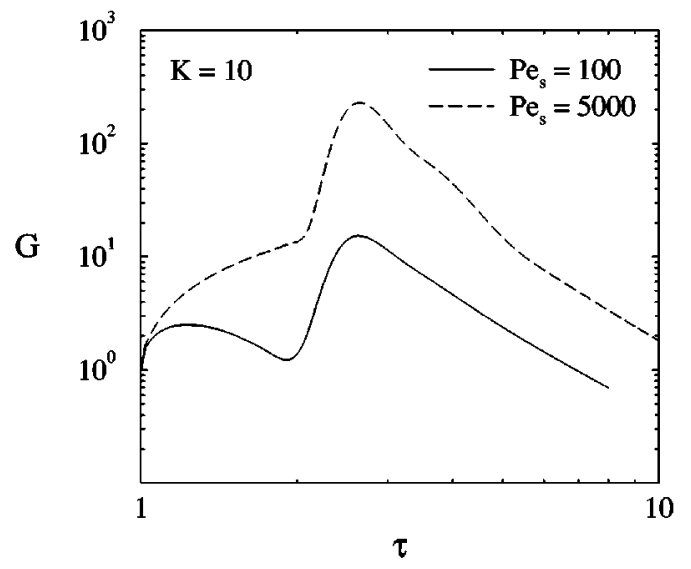

(b)

FIG. 9. The effect of decreasing $\mathrm{Pe}_{s}$ on the transient amplification and growth of disturbances. (a) Base state concentration gradient at time $\tau=2.6$ for $\mathrm{Pe}_{s}=100$ and 5000. (b) Amplification ratio for $\mathrm{Pe}_{s}=100$ and 5000. Relevant parameter values are $K=10, \xi_{s}$ $=2.0$, and $\mathcal{C}=10^{-5}$. Note that the smaller value of $\mathrm{Pe}_{s}$ eliminates the kink in $g_{o \xi}$ which reduces the amplification ratio by a considerable amount.

very surface active and therefore spread more rapidly along the surface of the liquid film. We repeated similar calculations as before and found the same qualitative behavior for transient amplification and growth as already described. Disturbances placed well ahead of the initial concentration decay point get significantly amplified when the advancing front meets and interacts with the applied perturbation. As these disturbances get convected behind the leading edge of the spreading film to the linear portion of the base-state concentration profile, they rapidly dissipate in energy and amplitude. Because monolayers with smaller values of $\beta$ are more surface active, the monolayer spreads more rapidly and the overall residence time of a disturbance in the forward part of the film is decreased. We observed a significant reduction in the amplification ratio $G(\tau)$ for values of $\beta$ of order one or less.

\section{CONCLUSION}

We have studied the evolution of linearized disturbances for a system consisting of a surfactant monolayer spreading

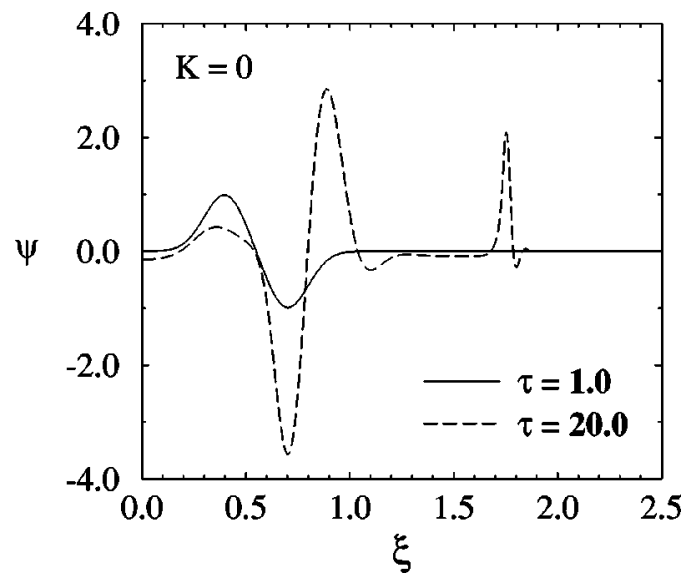

FIG. 10. The evolution of a global $\psi$ at $\tau=1.0$ and $\tau=20.0$ for $\mathrm{Pe}_{s}=5000, \mathcal{C}=10^{-5}$ and $K=0$.

on a thin viscous film. Large transient growth is achieved in cases where both the gradient of the surfactant concentration profile and the liquid height profile have time to develop their characteristic kink and shock at the leading edge of the distribution profile. These structures are very sensitive to disturbances and occur in the region of the spreading film, where the base state achieves a change in the direction of surface velocity. This reversal of fluid motion is directly due to the vanishing of the Marangoni force and the change in the capillary pressure at the advancing front. Consequently, surfactant and liquid will buildup at the monolayer edge creating the described behavior in the base states. Our studies indicate that if the base-states have sufficient time to evolve a shock front before encountering the disturbance, the perturbation essentially rides the wave supplied by the thickened front of the advancing film. Once the disturbances fall behind this advancing rim, they lose amplitude and energy and quickly decay to zero. Disturbances initially placed within the confines of the surfactant monolayer show little or no transient growth.

Leaving all relevant variables fixed and changing only the surface Peclet number lends insight into the mechanism responsible for disturbance enhancement. Lower Peclet numbers introduce stronger surface diffusion which smooths the kink in the concentration gradient profile as well as the advancing liquid shock. Once this region is smoothed, the amplification is all but eliminated. Although these localized disturbances are enhanced at the leading edge, they appear to dissipate before reaching the region of flow where the observed fingering occurs.

Preliminary results have shown that the application of more spatially extended disturbances capture two distinct characteristics of perturbation. Figure 10 shows an initial $\psi$ at $\tau=1$ and its development at a later time $\tau=20$ when $\mathrm{Pe}_{s}=5000, \mathcal{C}=10^{-5}$ and $K=0$. Upon application of this global disturbance, two distinct regions appear in $\psi$. Far downstream, a spike in $\psi$ develops at the leading edge of the monolayer. This appears to be the region characterized in this paper through the application of localized disturbances. However, in the upstream portion of the flow, a second re- 
gion of corrugations appear in $\psi$. This region of the disturbance bears a striking similarity to both the shape and location of the experimentally observed fingers. The analysis of this second region of the perturbations forms the basis of our next study.

\section{ACKNOWLEDGMENTS}

We gratefully acknowledge financial support for this study from the National Science Foundation through Grant Nos. CTS-9973538, CTS-0088774, and DMR-9809483.
[1] A. Marmur and M.D. Lelah, Chem. Eng. Commun. 13, 133 (1981).

[2] S.M. Troian, X.L. Wu, and S.A. Safran, Phys. Rev. Lett. 62, 1496 (1989).

[3] A.M. Pereira, B.S.E thesis, Princeton University, 1995.

[4] S. Zhu, W.G. Miller, L.E. Scriven, and H.T. Davis, Colloids Surf., A 90, 63 (1994).

[5] B. Frank and S. Garoff, Langmuir 11, 87 (1995).

[6] S. He and J.B. Ketterson, Phys. Fluids 7, 2640 (1995).

[7] S. Bardon, M. Cachile, A.M. Cazabat, X. Fanton, and S. Villette, Faraday Discuss. 104, 307 (1996).

[8] S. M. Troian, X.L. Wu, E. Herbolzheimer, and S.A. Safran, in Phase Transitions in Soft Condensed Matter, Vol. 211 of NATO Advanced Studies Institute, Series B: Physics, edited by T. Riste and D. Sherrington (Plenum Press, New York, 1989), p. 245.

[9] O.E. Jensen and J.B. Grotberg, J. Fluid Mech. 240, 259 (1992).

[10] B.F. Farrell and P.J. Ioannou, J. Atmos. Sci. 53, 2025 (1996).

[11] B.F. Farrell and P.J. Ioannou, J. Atmos. Sci. 53, 2041 (1996).

[12] O.K. Matar and S.M. Troian, Phys. Fluids 10, 1234 (1998).

[13] O.K. Matar and S.M. Troian, Phys. Fluids 11, 3232 (1999).

[14] O.K. Matar and S.M. Troian, Chaos 9, 141 (1999).

[15] F.F. Espinosa, Adv. Bioengineering ASME 20, 571 (1991).
[16] S.F. Shen, J. Aerosp. Sci. 28, 397 (1961).

[17] W.E. Schiesser, The Numerical Method of Lines (Academic Press, San Diego, 1991).

[18] A.C. Hindmarsh, in Scientific Computing, edited by R.S. Stepleman (North Holland, Amsterdam, 1983), p. 55.

[19] The representative values of $\mathrm{Pe}_{s}$ and $\mathcal{C}$ are chosen based on parameter values used in experiments. Typical values of the initial spreading pressure $\Pi^{*}$ and the surface diffusion coefficient $\mathcal{D}_{s}^{*}[23-25]$ are 10 dynes $/ \mathrm{cm}$ and $10^{-5} \mathrm{~cm}^{2} / \mathrm{sec}$. For a liquid viscosity of $\mu^{*}=10 \mathrm{cp}, \mathrm{Pe}_{s}=5000$ corresponds to an initial film thickness of $5 \mu \mathrm{m}$. With $\sigma_{m}^{*}=60 \mathrm{dyn} / \mathrm{cm}, \mathcal{C}$ $=10^{-5}$ corresponds to a value $\varepsilon \approx 0.001$, which satisfies the lubrication approximation.

[20] O.E. Jensen and D. Halpern, J. Fluid Mech. 372, 273 (1998).

[21] O.E. Jensen, J. Fluid Mech. 372, 301 (1998).

[22] D.P. Gaver and J.B. Grotberg, J. Fluid Mech. 213, 127 (1990).

[23] E.K. Sakata and J.C. Berg, Ind. Eng. Chem. Fundam. 8, 570 (1969).

[24] P.A. Good and R.S. Schechter, J. Colloid Interface Sci. 40, 99 (1972).

[25] M.L. Agrawal and R.D. Neuman, J. Colloid Interface Sci. 121, 366 (1988) 